\title{
Numerical Aspects of Spatio-Temporal Current Density Reconstruction from EEG-/MEG-Data
}

\author{
Uwe Schmitt, Alfred K. Louis*, Felix Darvas, Helmut Buchner, and Manfred Fuchs
}

\begin{abstract}
The determination of the sources of electric activity inside the brain from electric and magnetic measurements on the surface of the head is known to be an ill-posed problem.

In this paper, a new algorithm which takes temporal a priori information modeled by the smooth activation modell into account is described and compared with existing algorithms such as Tikhonov-Phillips.
\end{abstract}

Index Terms-Electronencephalography, inverse source reconstruction, magnetoencephalography, spatial-temporal current density reconstruction.

\section{INTRODUCTION}

I NVERSE source reconstruction has many applications in clinical and theorectical medicine. Examples are the noninvasive localization of focal epileptogenic discharges [1], [2] and the study of somato sensoric evoked potentials (SEPs) [3]. An overview of reconstruction methods is given in [4]. The same techniques can be applied to the human heart, e.g., in order to localize the origin of premature and extrasystolic beats [5].

Measurements of the electromagnetic activity of the brain with electroencephalography (EEG) or magnetoencephalography (MEG) provide an excellent temporal resolution, compared with positron emission tomography (PET) or functional magnetic resonance imaging (fMRI). EEG and MEG have a considerable lack of spatial resolution and are strongly affected by noise. As we will see, the problem of determining electrical sources inside the scalp from EEG/MEG-measurements is ill posed. Thus, we need additional spatial and temporal information about the brain activity in order to tackle this problem.

There are two different strategies for the solution of the inverse problem.

- By optimization techniques a small number of dipoles and their parameters (location, orientation and magnitude) are calculated ensuring best fit between measured data

Manuscript received November 6, 2000; revised February 5, 2001. This work was supported by the German Ministry of Education and Research under Grant 03-LO7SA2-2, 03-BU7AA1-4. The Associate Editor responsible for coordinating the review of this paper and recommending its publication was M. W. Vannier. Asterisk indicates corresponding author.

U. Schmitt is with the Institute of Applied Mathematics, Universitaet des Saarlandes, 66041 Saarbruecken, Germany.

*A. K. Louis is with the Institute of Applied Mathematics, Universitaet des Saarlandes, 66041 Saarbruecken, Germany (e-mail: louis@ @um.uni-sb.de).

F. Darvas is with the Department of Neurology, RWTH Aachen, 52057 Aachen, Germany.

H. Buchner is with the Clinics for Neurology and clinical Neurophysiology, 45667 Recklinghausen, Germany.

M. Fuchs is with the Neuroscan Labs, 20255 Hamburg, Germany.

Publisher Item Identifier S 0278-0062(01)03549-2. and forward calculated potentials, respectively, magnetic fluxes [6], [7]. This is a nonlinear problem and regularization is achieved by the small number of parameters. An important problem is the correct estimation of the number of dipoles. Also, in general, only the cross product of location and moment of dipole is determined [17].

- Alternatively, in the current density reconstruction (CDR) the distribution of the currents is determined. Being an under-determined problem further information about the currents is needed, introduced as additional constraints. One possible constraint is the minimum norm criterion [8], [9]. Current CDR procedures reconstruct sources seperatly for each time slice. We present a new CDR method by introducing a temporal constraint, the smooth temporal activation model. This constraint also achieves reconstructions with a deeper insight into the time dynamics of the sources. CDR techniques lead in some cases to linear problems. For a survey of different CDR techniques, see [10]. CDR methods are getting more and more important in EEG/MEG-source localization practice since the estimation of the number of unknown dipoles in dipole-fit methods is problematic.

\section{THE EEG/MEG ForwARD MODEL}

The fundamental equation governing the interaction of electrical sources $j$ and the electrical field $\Phi$ is the Poisson equation in connection with a Neumann boundary condition

$$
\begin{aligned}
\operatorname{div}(\sigma \nabla \Phi) & =\operatorname{div} j \quad \text { in } \Omega \\
\langle\sigma \nabla \Phi, n\rangle & =0 \quad \text { at } \Gamma=\partial \Omega .
\end{aligned}
$$

Here, $\sigma$ is the conductivity tensor and the open and bounded set $\Omega$ describes the geometry of the head. $n$ is the outward normal at $\partial \Omega$.

For the following discussion, we have to introduce the so-called Sobolev spaces $H^{1}$ and $H_{0}^{1}$. These are Hilbert spaces of functions which are differentiable in a generalized sense where the first derivatives are square integrable. The functions of $H_{0}^{1}$ additionally have zero boundary values. For a formal definition, see [11] or [12]. These Sobolev spaces are well suited to modell current-densities. Pure dipoles do not belong to these function spaces.

We define $\Gamma_{0} \subset \Gamma$ as the EEG measurement surface and $\Gamma_{1}$ such that $\Gamma_{1} \cap \bar{\Omega}=\emptyset$ as the MEG mesurment surface.

The EEG forward model can be specified as follows:

$$
\begin{aligned}
A_{E}:\left(H_{0}^{1}(\Omega)\right)^{3} \stackrel{\operatorname{div}}{\longrightarrow} L^{2}(\Omega) \stackrel{S}{\longrightarrow} H^{1}(\Omega) \stackrel{\gamma}{\longrightarrow} L^{2}\left(\Gamma_{0}\right) \\
j \longmapsto J=\operatorname{div} j \longmapsto \Phi \longmapsto \Phi \mid \Gamma_{0} .
\end{aligned}
$$


Here, $S$ is the weak solution operator of (1), $\gamma$ is the trace operator from $\Omega$ to $\Gamma_{0}$ [12], [13].

We define

$$
(T f)(x)=\int_{\Omega} f(y) \times \frac{x-y}{|x-y|^{3}} d y
$$

According to the Biot-Savart law the MEG-measurements are modeled by

$$
B(x)=\left\langle T(j+\sigma \nabla \Phi)(x), n_{x}\right\rangle
$$

with $x \in \Gamma_{1}$ and $n_{x}$ is the outward normalvector in $x$. Thus, the MEG forward model can be described as

$$
\begin{gathered}
A_{M}:\left(H_{0}^{1}(\Omega)\right)^{3} \stackrel{S \text { div }}{\longrightarrow} H^{1}(\Omega) \stackrel{j+\sigma \nabla \cdot}{\longrightarrow}\left(L^{2}(\Omega)\right)^{3} \stackrel{\left\langle T \cdot, n_{x}\right\rangle}{\longrightarrow} L^{2}\left(\Gamma_{1}\right) \\
j \longmapsto \Phi \longmapsto j+\sigma \nabla \Phi \longmapsto B .
\end{gathered}
$$

Theorem 1: The operators

$$
A_{E}:\left(H_{0}^{1}(\Omega)\right)^{3} \rightarrow L^{2}\left(\Gamma_{0}\right)
$$

and

$$
A_{M}:\left(H_{0}^{1}(\Omega)\right)^{3} \rightarrow L^{2}\left(\Gamma_{1}\right)
$$

are compact and have nontrivial nullspaces.

Proof: The trace operator $\gamma$ is compact for the given spaces [13]. $T$ is compact, as the kernel in (3) is continuous, and $\Gamma_{1} \cap \bar{\Omega}=\emptyset$. The remaining operators in (2) and (5) are continuous [13]. Thus, $A_{M}$ and $A_{E}$ are compact. As the divergence operator has a nontrivial nullspace, the same is valid for $A_{E}$ and $A_{M}$.

Corollary 2: The problems

$$
A_{E} j=\Phi_{0}, \quad \text { respectively, } A_{M} j=B_{0}
$$

and the combined problem

$$
A_{E} j=\Phi_{0} \wedge A_{M} j=B_{0}
$$

are ill posed.

Here, "ill posed" means that the involved operators are not continously invertible which has the consequence that noise in the data has strong effects on the reconstruction results.

\section{THE LEADFIELD MATRIX}

To discretize the problem we represent the current in $\Omega$ as a sum of a fixed number of dipoles, located at points $p_{i}$ in $\Omega, 1 \leq$ $i \leq N$, and we use point collocation at the measurement points. The set $\left\{p_{i} \mid 1 \leq i \leq N\right\}$ is called influence space.

It is assumed that measurements are taken at $n$ points $\xi_{i}$ on $\Gamma_{0} \cup \Gamma_{1}$. The so-called leadfield matrix $L \in \mathbb{R}^{n \times 3 N}$ contains in the $i$ th column the data vector $m_{i}$ of size $n$ belonging to a dipole with moment $(1,0,0)^{\top}$ located at influence point $p_{i}$. Columns $i+N$ and $i+2 N$ contain data belonging to dipoles with moments $(0,1,0)^{\top}$ and $(0,0,1)^{\top}$, respectively. In detail, if we define $\bar{e}_{k}$ as the $k$ th unit-vector and $e_{i, k}$ as a dipole with the moment $\bar{e}_{k}$ located at point $p_{i}$ we get

$$
L_{\nu, i+k N}=A_{M / E} e_{i, k}\left(\xi_{\nu}\right) .
$$

If we now discretize $\mathbf{j}$ by $\mathbf{j}=\sum_{i=1}^{N} \sum_{k=0}^{2} j_{i+k N} e_{i k}$, we get

$$
\begin{aligned}
A_{M / E} \mathbf{j}\left(\xi_{\nu}\right) & =\sum_{i=1}^{N} \sum_{k=0}^{2} j_{i+k N} A_{M / E} e_{i k}\left(\xi_{\nu}\right) \\
& =\sum_{i=1}^{N} \sum_{k=0}^{2} j_{i+k N} L_{\nu, i+k N} \\
& =(L j)_{\nu} .
\end{aligned}
$$

Thus, the product $L j$ yields the data $m$ belonging to the disretized current distribution $\mathbf{j}$.

In practice, $\Omega$ and $\sigma$ can be obtained from MRI pictures. The columns $(L)_{, i}$ of $L$ are then computed by the finite element method according to [14]. For isotropic $\sigma$, the boundary element method can be used too [15]. Analytical formulas are only available for simplified geometries as, e.g., concentric ellipses or spheres [16], [17].

\section{Conventional CDR Methods}

As we already mentioned source reconstruction methods are classified as dipole reconstruction methods and current reconstruction methods. The first one tries to determine $q$ dipoles at locations $p_{i}$ with moment $M_{i}$ and unknown $q$ such that this configuration explains the measured data. This leads to a nonlinear optimization problem. Current reconstruction methods (CDR methods) try to determine $j$ from data $m$. The problem $L j=m$ is a linear problem.

In practice, the number of influence points $N$ is much larger than the number of measurements $n$. Thus, the system $L j=m$ is underdetermined. Due to the ill posedness of the problem, the matrix $L$ is ill conditioned. Thus, we need additional a priori information to achieve a unique and stable solution. One possibility is Tikhonov-Phillips regularization [14], [18], [19]

$$
j=\operatorname{minarg}\left\{\|L j-m\|_{p}^{p}+\lambda^{2}\|T j\|_{q}^{q}\right\} .
$$

$T$ is called spatial model operator. Due to the large range of the norms of the columns of $L$ one has to use a depth-weighting matrix $W$ in order to get bias-free reconstructions (see [10]). $p=q=2, T=W$ yields minimum norm least squares solutions, $p=q=2, T=\triangle W$ is in this field of application the so called LORETA method [21]. In the first case, one achieves so called zero-order spatial smoothness, in the second case one achieves second order spatial smoothness. $\triangle$ is a discrete version of the Laplace operator $\Delta$. For an implementation of $\Delta$, see [20]. Both cases lead to linear problems, due to $p=q=2$.

This approach does not take temporal information into consideration. Calculations only use one timeslice of data.

\section{Spatio-Temporal CDR: The SMOOTh Activation MODEL}

In this section, we assume the data $m$ are given as a function of time $m(t)$. Hence the solution $j(t)$ of (1) is also a function of time. Notice that the model is assumed to be stationary, i.e., $\sigma$ is not a function of time. So we can apply the methods discussed in Section IV for each time instance.

A coupling of different time instances is achieved by the assumption that the electrical sources do behave temporally 


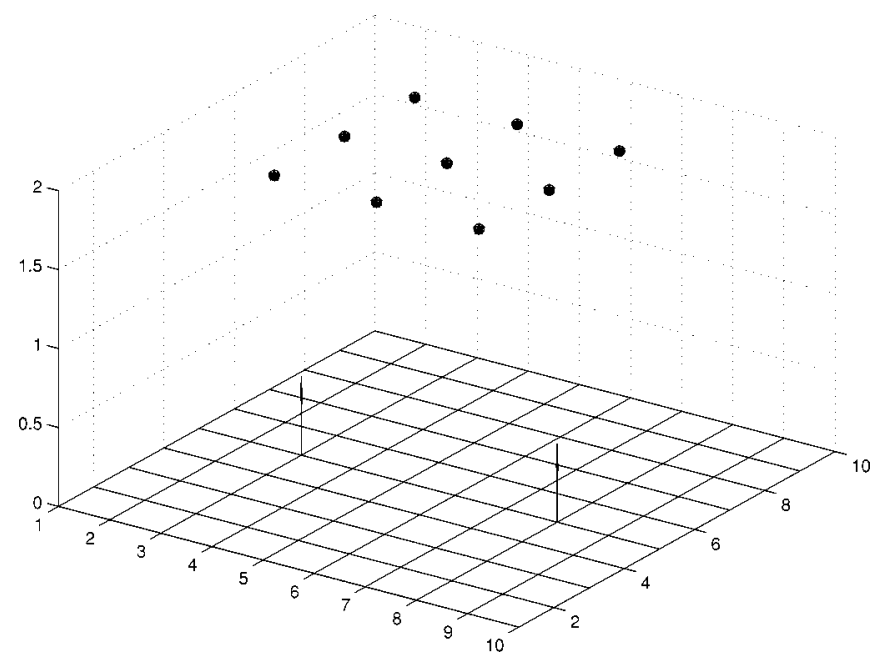

Fig. 1. Sketch of the simple problem.

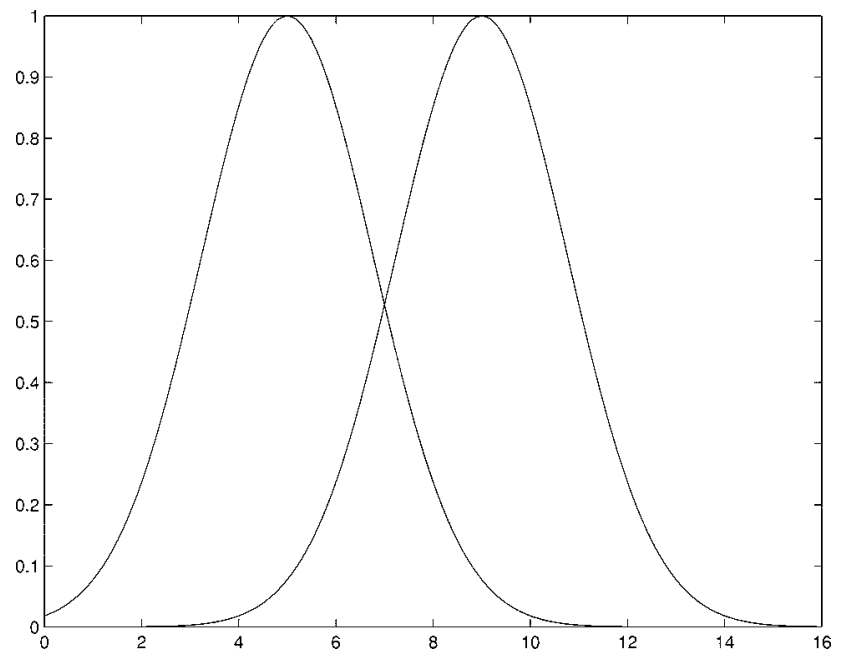

Fig. 2. The activation curves of the dipoles.

smoothly. Hence we make the pysiologically motivated assumption

$$
\left\|\frac{d}{d t} j(t)\right\| \rightarrow \min .
$$

Assume that the data $m_{l}$ are given for $T$ timeslices $t_{l}$. The current at time $t_{l}$ is termed as $j_{l}$.

In order to achieve a temporal coupling according to (7), one approximates

$$
\frac{d}{d t} j(t) \approx \frac{1}{\Delta t}(j(t+\Delta t)-j(t))
$$

and we get an additional model term which extends the minimization problem (6) in the following way:

$$
\begin{aligned}
\left(j_{1}, \ldots, j_{T}\right)=\operatorname{minarg}\{ & \left\{\sum_{i=1}^{T}\left\|L j_{i}-m_{i}\right\|_{2}^{2}+\lambda^{2} \sum_{i=1}^{T}\left\|T j_{i}\right\|^{2}\right. \\
& \left.+\mu^{2} \sum_{i=1}^{T-1}\left\|j_{i+1}-j_{i}\right\|_{2}^{2}\right\}
\end{aligned}
$$

This is the model of the spatio-temporal CDR method.

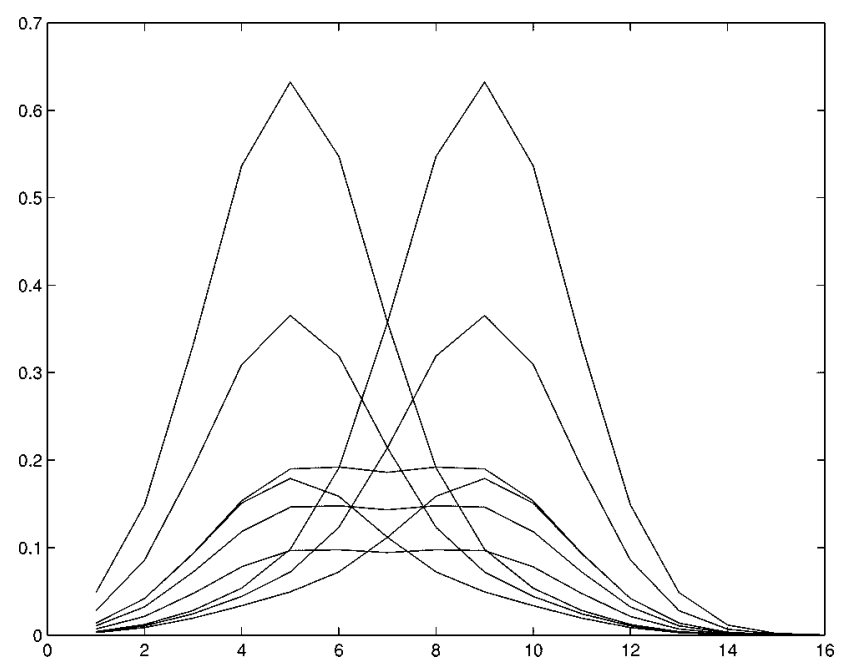

Fig. 3. Synthetic data.

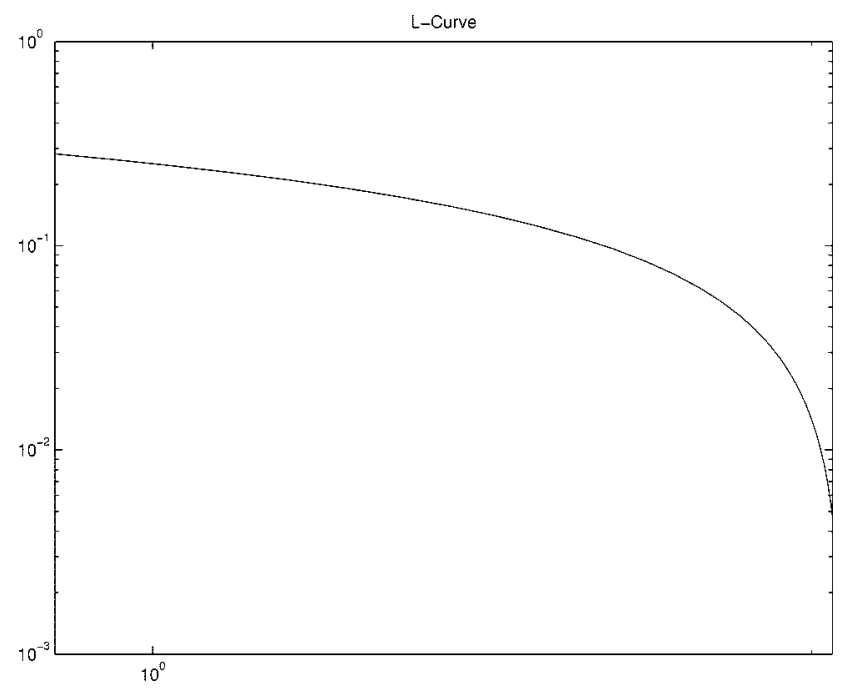

Fig. 4. $L$-curve for the determination of $\lambda$.

We introduce the so called kronecker product by

$$
A \otimes B:=\left[\begin{array}{ccc}
a_{1,1} B & \cdots & a_{1, m} B \\
\vdots & & \vdots \\
a_{n, 1} B & \cdots & a_{n, m} B
\end{array}\right] .
$$

Now (8) can be formulated as follows:

$$
\mathbf{j}=\operatorname{minarg}\left\{\left\|L_{T} \mathbf{j}-\mathbf{m}\right\|_{2}^{2}+\lambda^{2}\|B \mathbf{j}\|_{2}^{2}+\mu^{2}\|K \mathbf{j}\|_{2}^{2}\right\} .
$$

Here

$$
\begin{aligned}
\mathbf{j} & =\left(j_{1}, \ldots, j_{T}\right)^{\top} \\
\mathbf{m} & =\left(m_{1}, \ldots, m_{T}\right)^{\top} \\
L_{T} & =I_{T} \otimes L=\operatorname{blockdiag}(L, \ldots, L) \\
K & =D \otimes I_{3 N} \\
& =\left[\begin{array}{cccc}
I_{3 N}-I_{3 N} & 0 & \ldots & 0 \\
0 & I_{3 N}-I_{3 N} & \cdots & 0 \\
\vdots & \ddots & & \vdots \\
0 & \ldots & 0 & I_{3 N}-I_{3 N}
\end{array}\right]
\end{aligned}
$$


1

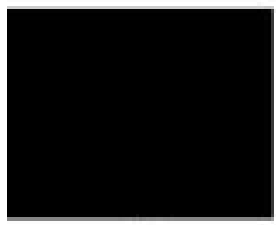

5

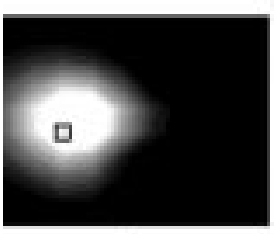

9

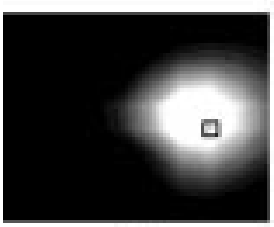

13

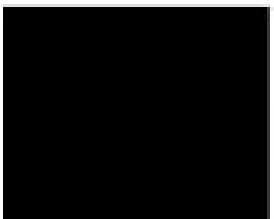

2

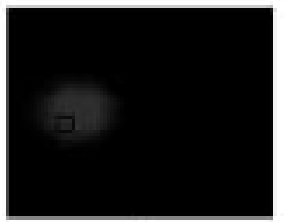

6

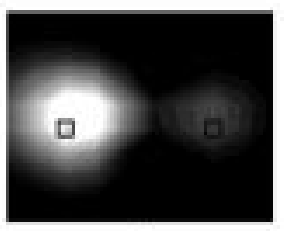

10

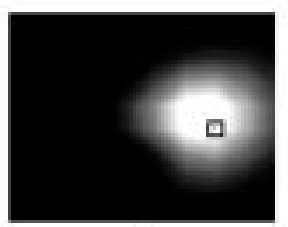

14

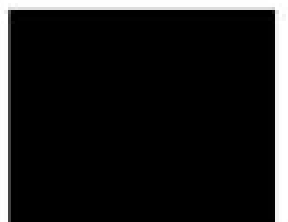

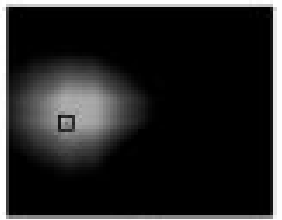

7

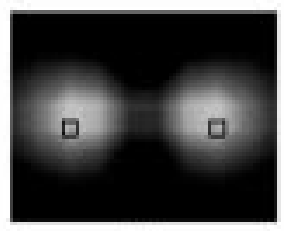

11

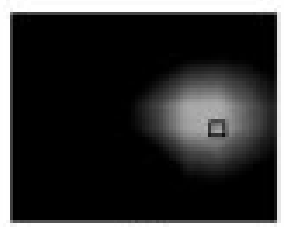

15

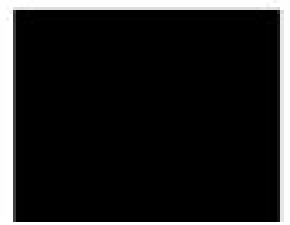

4

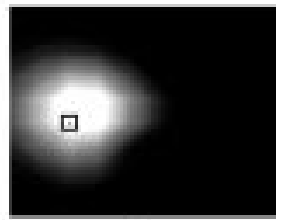

8

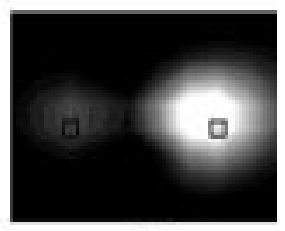

12

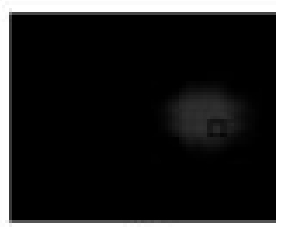

16

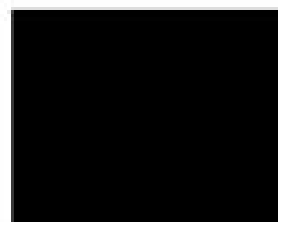

Fig. 5. Temporal reconstruction. Zeroth-order spatial smoothness and temporal coupling: $\lambda^{2}=0.01, \mu^{2}=0.2$. No noise added to the data.

$$
D=\left[\begin{array}{ccccc}
1 & -1 & 0 & \cdots & 0 \\
0 & 1 & -1 & \cdots & 0 \\
\vdots & & \ddots & & \vdots \\
0 & \cdots & 0 & 1 & -1
\end{array}\right]
$$

$B$ can be chosen as

$$
B=I_{T} \otimes W=\operatorname{blockdiag}(W, \ldots, W)
$$

or

$$
B=I_{T} \otimes(\triangle W)=\operatorname{blockdiag}(\triangle W, \ldots, \triangle W) .
$$

Due to $\nabla\|A x-b\|_{2}^{2}=2\left(A^{\top} A x-A^{\top} b\right)$, (9) leads to the regularized normal equation

$$
\left(L_{T}^{\top} L_{T}+\lambda^{2} B^{\top} B+\mu^{2} K^{\top} K\right) \mathbf{j}=L_{T}^{\top} \mathbf{m} .
$$

The matrix at the left hand side is positive definite, so an efficient way to solve the problem is the cg-algorithm. In practical applications, the whole matrix on the left side of (10) is too big to fit into memory (a typical size for $L$ is $64 \times 10^{4}$ ), but the cg-algorithm only needs the evaluation of the multiplication of the matrix above by an arbitrarily given vector. So one only has to keep the matrices $L$ and $K$ in memory. Due to the size of $L$ this solver is quite slow. A remarkable improvement can be achieved in some special cases. We will discuss this in the Appendix.

\section{Simulations}

\section{A. The Simple Volume Conductor Model}

For comparing the properties of the methods (6) and (9), a simple volume conductor model was chosen: we consider a three-dimensional (3-D) problem with a two-dimensional (2-D) influence space. This setup is nearer to reality than a 2-D problem with a one-dimensional influence space and allows an easier comparision of reconstruction results than a real 3-D influence space.

The simple model set up is made up of an influence space consisting of a $10 \times 10$ grid with a length of ten arbitrary units per side centered at $(5.5,5.5,0.0)$. Nine sensors were placed in a square planar array with center at $(5.5,5.5,2)$ above the grid. See Fig. 1.

We use constant conductivity $\sigma$ inside and outside the object. Thus, the leadfield matrix is obtained by

$$
\begin{aligned}
L_{i, j} & =\frac{1}{4 \pi \sigma} \frac{r_{i, 1}-p_{j, 1}}{\left|r_{i}-p_{j}\right|^{3}} \\
L_{i, j+N} & =\frac{1}{4 \pi \sigma} \frac{r_{i, 2}-p_{j, 2}}{\left|r_{i}-p_{j}\right|^{3}} \\
L_{i, j+2 N} & =\frac{1}{4 \pi \sigma} \frac{r_{i, 3}-p_{j, 3}}{\left|r_{i}-p_{j}\right|^{3}} .
\end{aligned}
$$

Here, $r_{i} \in \mathbb{R}^{3}$ is the position of the $i$ th sensor, $p_{j} \in \mathbb{R}^{3}$ is the position of the $j$ th gridpoint. 
1

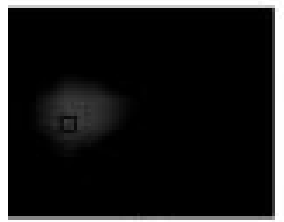

5

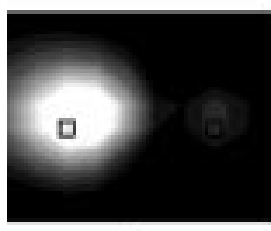

9

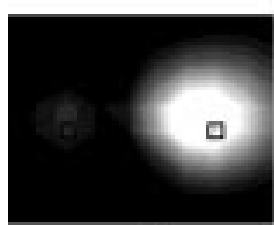

13

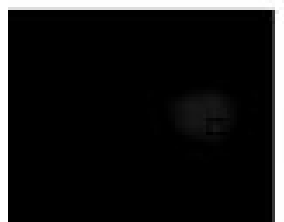

2

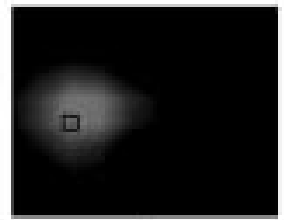

6

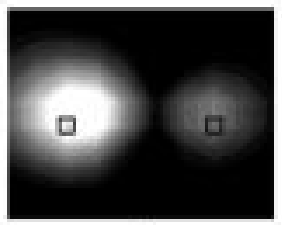

10

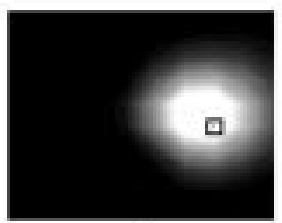

14

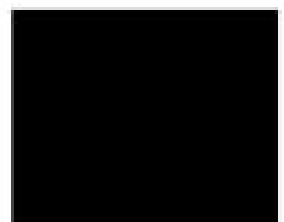

3

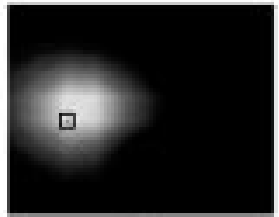

7

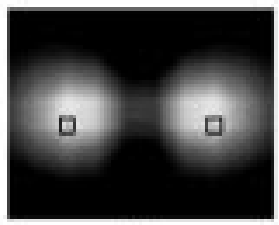

11

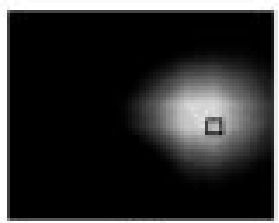

15

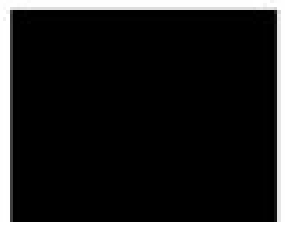

4

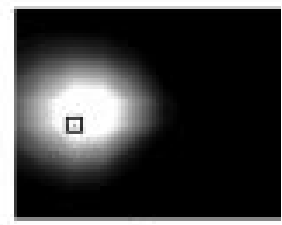

B

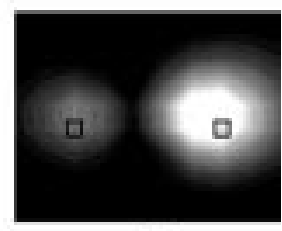

12

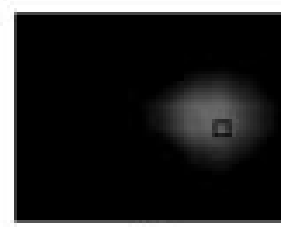

16

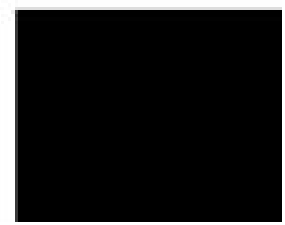

Fig. 6. Temporal reconstruction. Zeroth-order spatial smoothness without temporal coupling, $\lambda^{2}=0.01$. No noise added to the data.

\section{B. Inverse Calculations}

Two equally oriented dipoles with moment $(0,0,1)^{\top}$ at $x=$ $3, x=8$ and both at $y=5$ were placed on the ten by ten grid. These dipoles are drawn in Fig. 1. A gaussian dipole-strength time series was assigned to each dipole by

$$
q(t)=q_{0} \exp \left\{-\frac{\left(t-t_{p}\right)^{2}}{w^{2}}\right\}
$$

with peaks at timeslice 5 (dipole 1) and 9 (dipole 2) and a width of $w=2.5$. See Fig. 2 .

The simulations were calculated for 16 timeslices. The synthetic data are plotted in Fig. 3.

We used the timestep appendant to data with maximal amplitude for the determination of the regularization parameter $\lambda$ by the $L$-curve criterion for fixed error level ([22], [23]) applied to (6) with $p=q=2$. See Fig. 4.

For the solution of the normal (10), we used a cg-solver as proposed above. One important point concerning the runtime of such a solver is the choice of the initial value jo. We used the solutions of the uncoupled (6). In a simulation run with a big influence space, we achieved a solution of (10) after 19 iterations instead of 150 in the case of jo $=0$.

Figs. 5, 6, and 8-12, 16 show the contour-plots of $\|j(p, t)\|$. The white squares in the contour-plots indicate the original source-positions.

In Figs. 5 and 6, pure synthetic data were used to compare temporaly coupled and temporaly uncoupled solutions. The

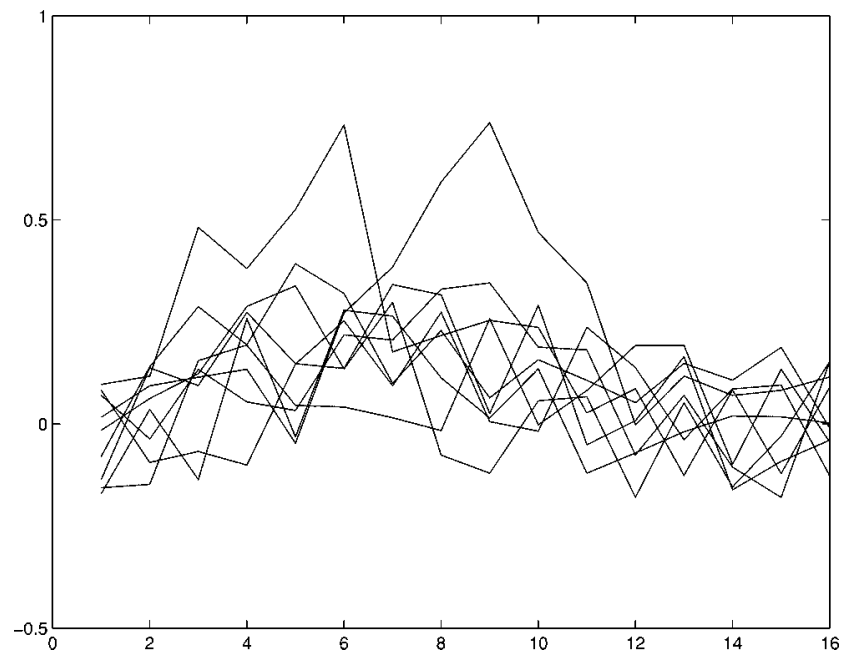

Fig. 7. Synthetic data with $30 \%$ noise added.

temporaly coupled solution seems to yield a little better spatial accuracy.

In the further simulations, uniform noise with $30 \%$ of the maximal signal amplitude was added to the data. See Fig. 7.

In Figs. 8 and 9, we compare temporaly coupled and noncoupled LORETA-solutions. The coupled solutions are significantly less affected by noise. In the time range from timeslice four to timeslice ten, the coupled solutions also reconstruct the correct number of sources, whereas the noncoupled solutions reconstruct only one source. 
$t=1$

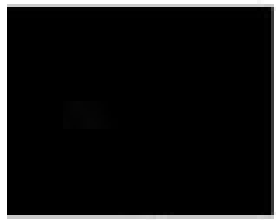

$\mathrm{t}=5$

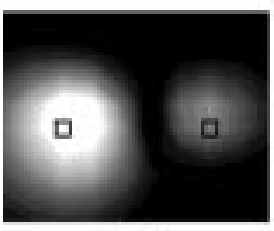

$\dagger=9$

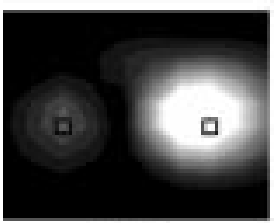

t=13

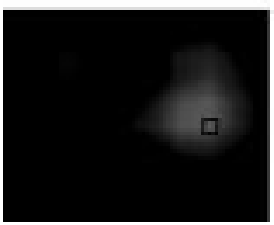

$t=2$

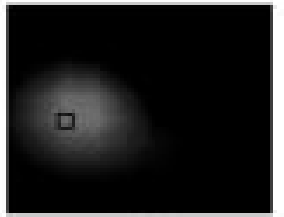

$t=6$

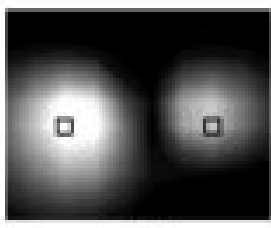

$t=10$

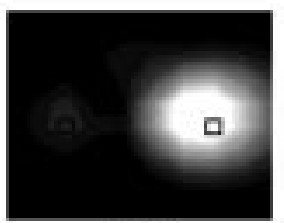

$t=14$

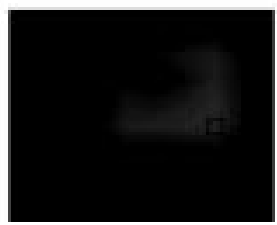

$t=3$

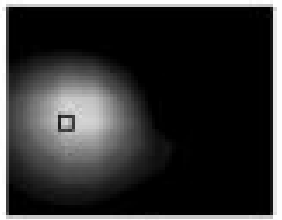

$\mathrm{t}=7$

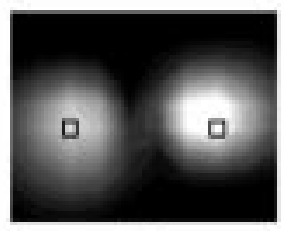

$t=11$

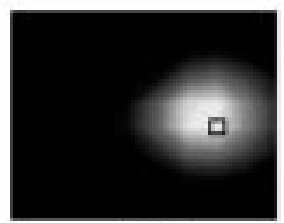

$t=15$

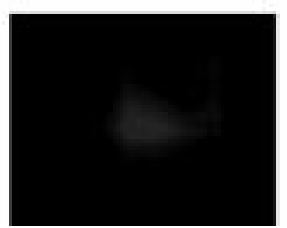

$t=4$

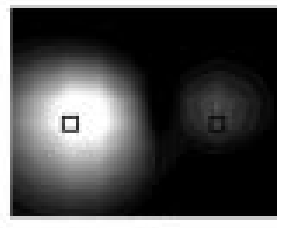

$t=8$

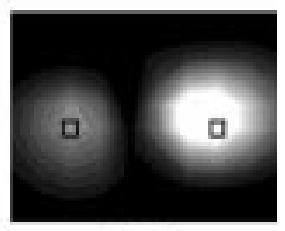

$t=12$

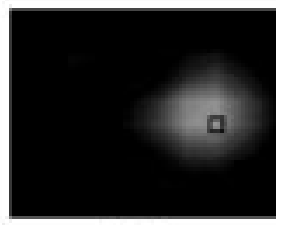

$t=16$

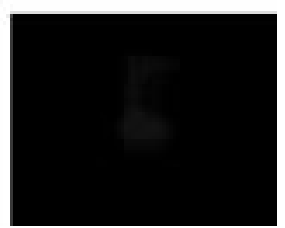

Fig. 8. Temporal reconstruction. Second-order spatial smoothness (LORETA) and temporal coupling: $\lambda^{2}=2.0, \mu^{2}=1.5$. Thirty percent noise added to the data.

$t=1$

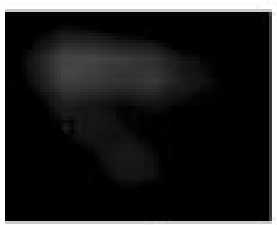

$t=5$

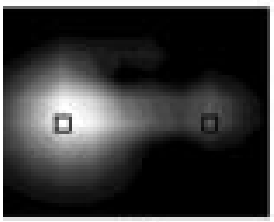

$t=9$

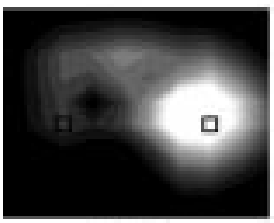

t -13

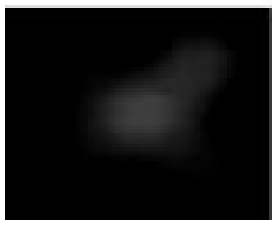

$t-2$

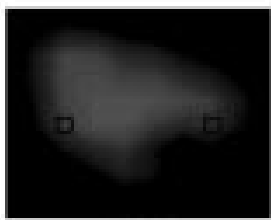

$t=6$

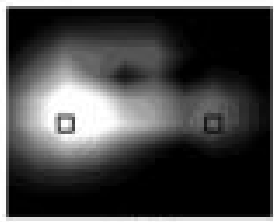

$t=10$

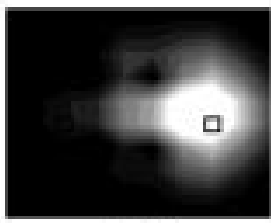

$t=14$

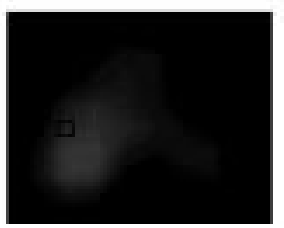

$t-3$

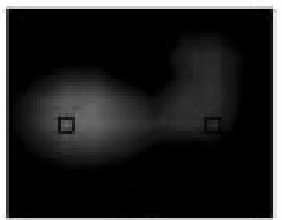

$t=7$

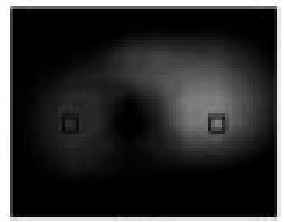

$t=11$

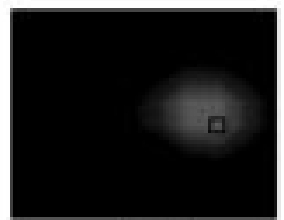

$\mathrm{t}=15$

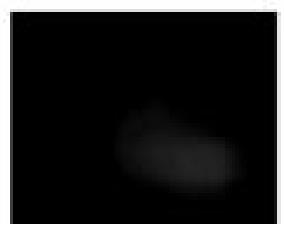

$t-4$

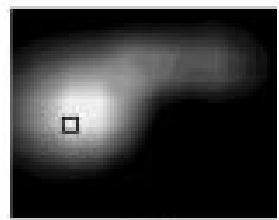

$\tan 8$

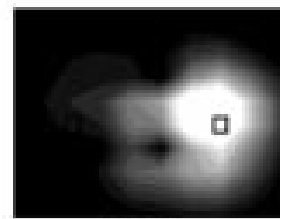

$t=12$

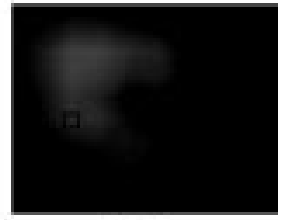

$t=16$

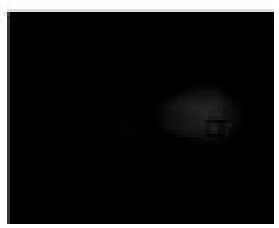

Fig. 9. Temporal reconstruction. Second-order spatial smoothness (LORETA) without temporal coupling: $\lambda^{2}=2.0$. Thirty percent noise added to the data. 


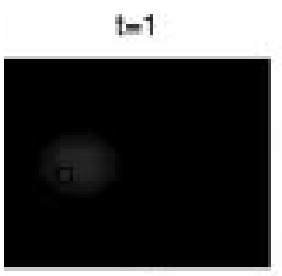

$t=5$

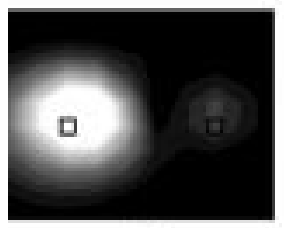

$t=9$

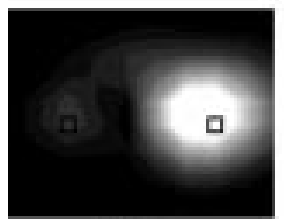

t=13

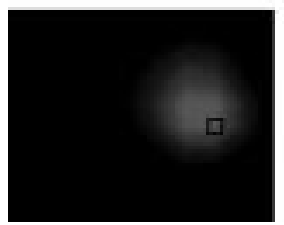

$t=2$

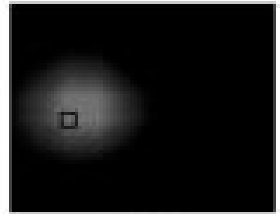

$t=6$

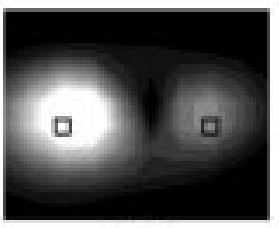

$t=10$

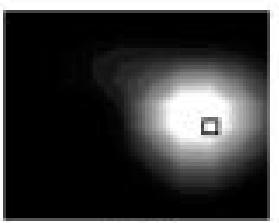

t=14

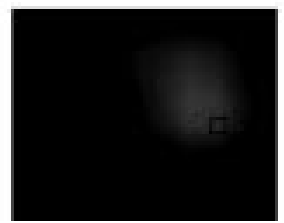

$t-3$

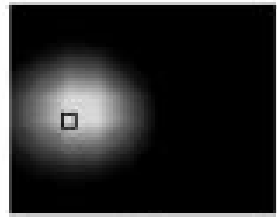

$\mathrm{t}=7$

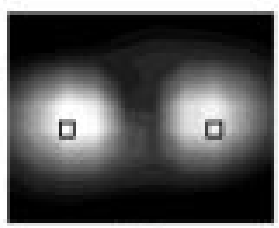

$t=11$

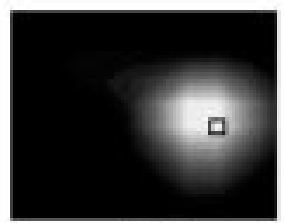

$t=15$

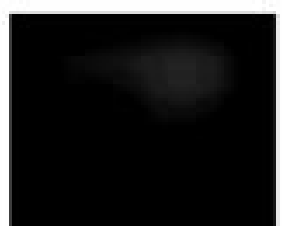

$t-4$

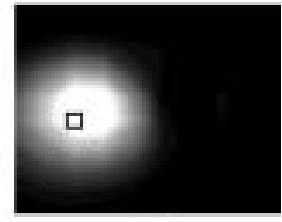

$t=8$

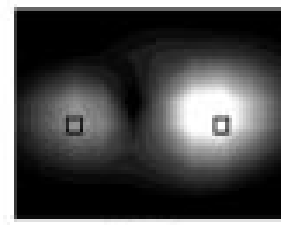

$\mathrm{t}=12$

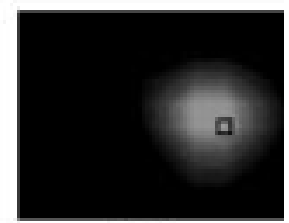

$t=16$

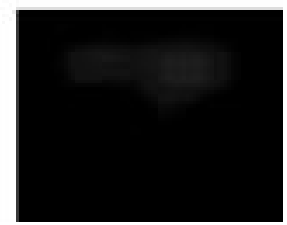

Fig. 10. Temporal reconstruction. Zeroth-order spatial smoothness and temporal coupling: $\lambda^{2}=2.0, \mu^{2}=1.5$. Thirty percent noise added to the data.

1

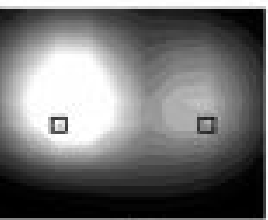

5

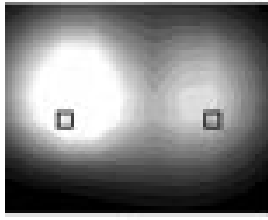

9

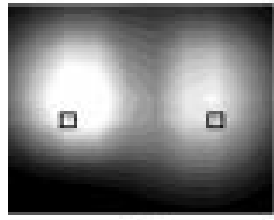

13

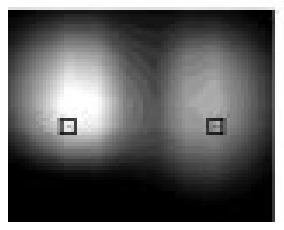

2

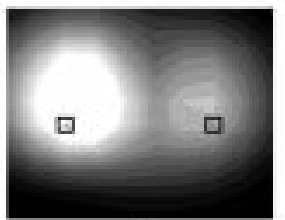

6

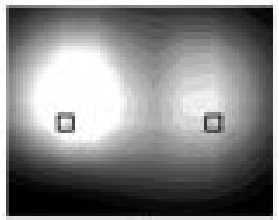

10

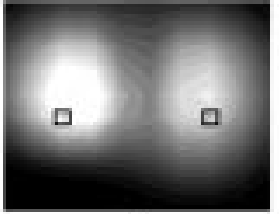

14

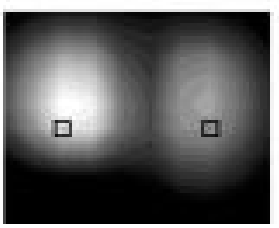

3

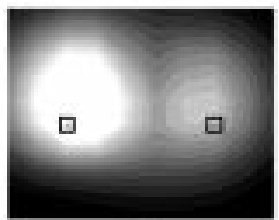

7

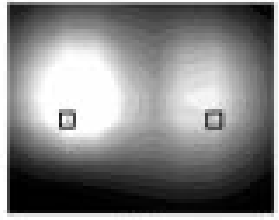

11

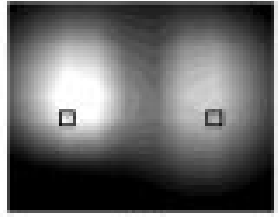

15

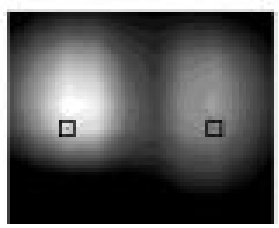

4

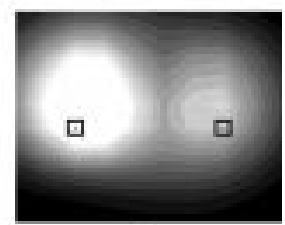

B

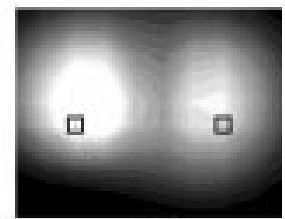

12

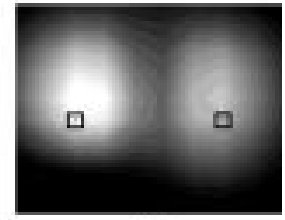

16

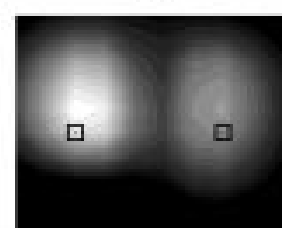

Fig. 11. Temporal reconstruction. Zeroth-order spatial smoothness and temporal coupling: $\lambda^{2}=2.0, \mu^{2}=15$. This demonstrates the effect of a strong temporal coupling. Thirty percent noise added to the data. 
$t=1$

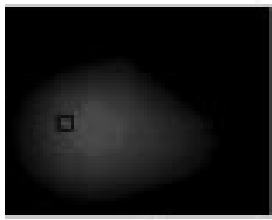

$t=5$

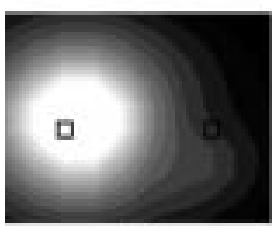

$t=9$

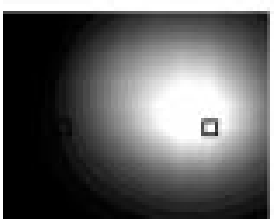

t -13

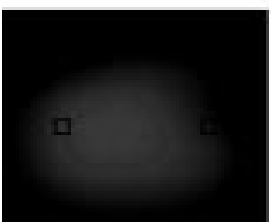

$t=2$

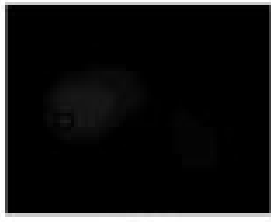

$t=6$

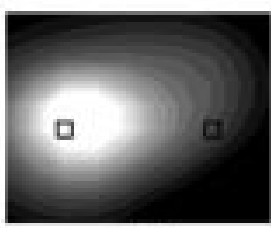

$t=10$

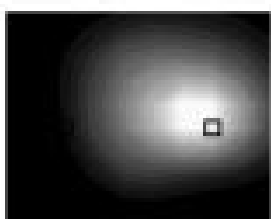

t=14

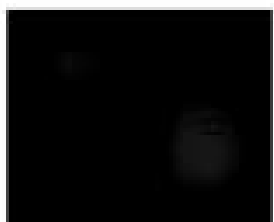

$t-3$

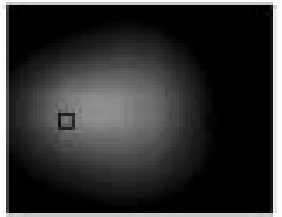

$t=7$

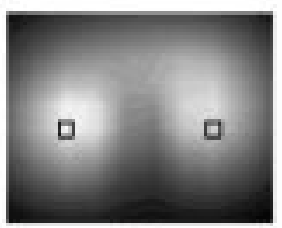

$t=11$

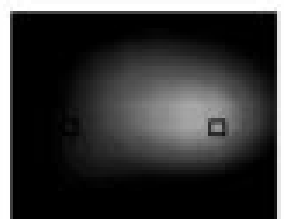

$t=15$

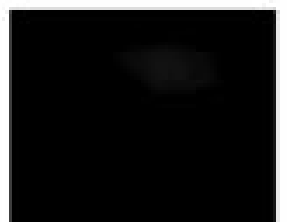

$t-4$

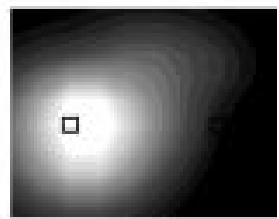

$t=8$

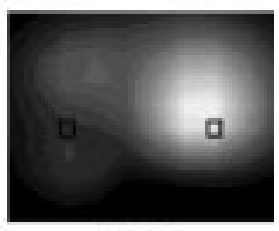

$t=12$

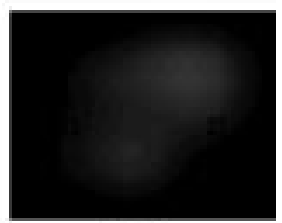

$t=16$

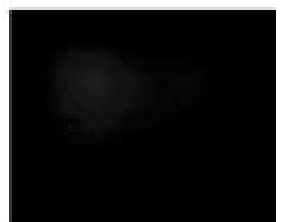

Fig. 12. Temporal reconstruction. Zeroth-order spatial smoothness without temporal coupling: $\lambda^{2}=2.0$. Thirty percent noise added to the data.

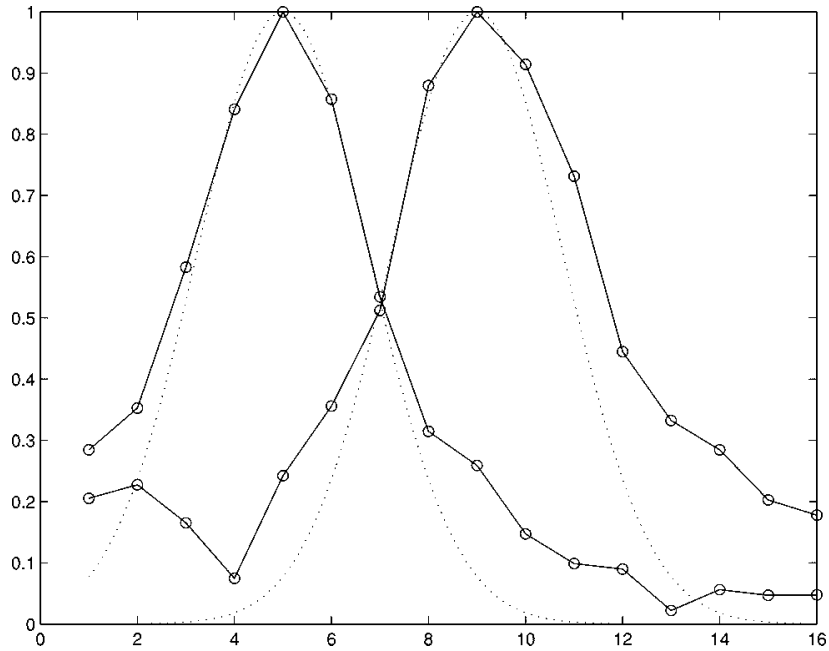

Fig. 13. Activation curve of temporal reconstruction with temporal coupling. $\lambda^{2}=2.0, \mu^{2}=1.5$. Thirty percent noise added to the data.

In Figs. 10-12, we see temporaly coupled and noncoupled zeroth-order smoothness solutions. In Fig. 11, we used $\mu=15$ in order to demonstrate the effects of a strong temporal coupling. Again the temporaly coupled solution in Fig. 10 is less affected by noise than the uncoupled solution in Fig. 12. Due to the second-order smoothness, the results in Fig. 8 are a bit smoother than the results in Fig. 10.

In Figs. 13 and 14, we draw the activation curves of temporaly coupled and uncoupled solutions. For these plots, we used

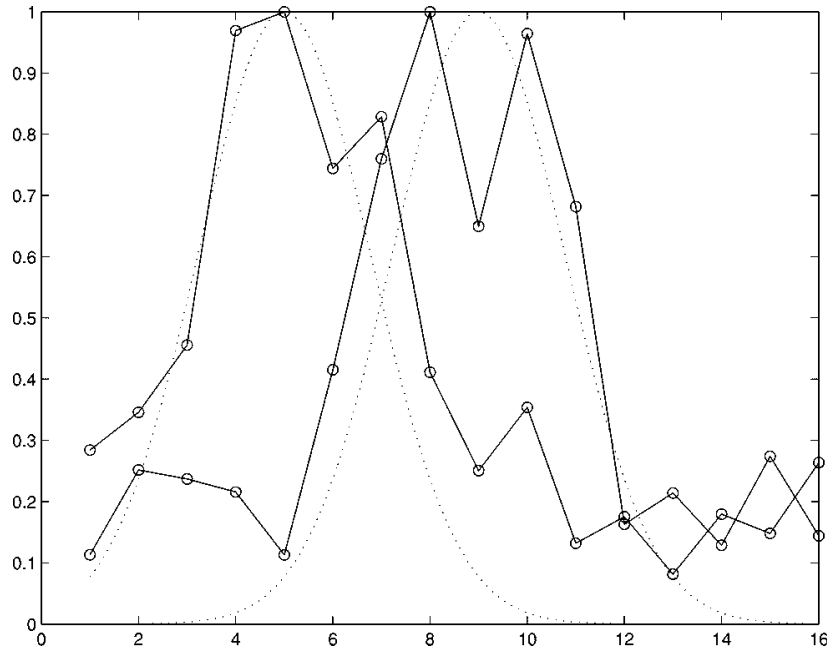

Fig. 14. Activation curve of temporal reconstruction without temporal coupling. $\lambda^{2}=2.0$. Thirty percent noise added to the data.

the activity at the local maxima of the contour plots above. As wecan see, the coupled solution gives a deeper insight into the time dynamics of the sources.

At last we used another method for temporal reconstructions: we smoothed the data with a Savitzky-Golay filter (also called "least squares filter") of order three and length five (see [24] and [25]). In Fig. 15, we see the smoothed data. Then we used these data to get a temporaly uncoupled solution. The results in Fig.16 are better than in the unsmoothed case but not as good as the results obtained by temporal coupling. 


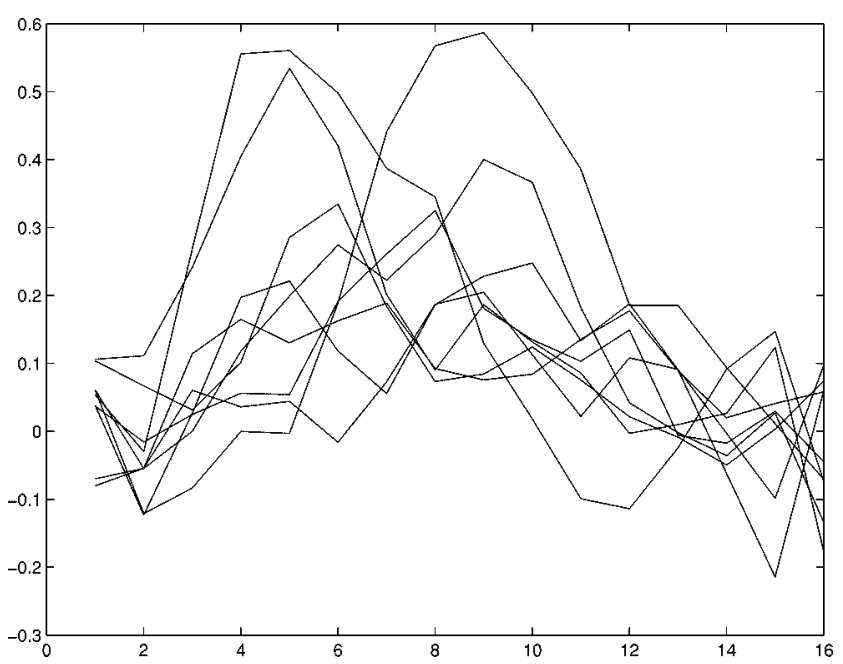

Fig. 15. Noisy data smoothed with a Savitzky-Golay filter.

For a systematic comparison of coupled and noncoupled solutions, we introduced two measures for the accuracy of the solutions.

- We compared the distance of the exact locations with the local maxima of the reconstructions. The dependency of this measure on the noise level is depicted in Fig. 17.

- We calculated the correlation of the true activation curve and the activation curves calculated from reconstructions. In Fig. 18, we see the dependency on the noise level.

\section{SUMMARY}

It could be shown in simulations, that the introduction of temporal constraints to existing CDRs leads to significant improvements in spatial and temporal accuracy. This general advantage of spatio-temporal CDR was shown to be most prominent in the case of noisy data, but also in the ability to seperate sources.

\section{APPENDIX}

In the following, we will consider two minimization problems which allow a very fast and efficient solution. These problems approximate problem (9).

- We introduce the first problem by ommitting the weighting matrix $W$ in problem (9). This is warrantable if we know a priori that the distance of the considered influence space to the sensors varies only little. For example, if we search sources on a part of the cortex which is nearly planar and parallel to the sensors. In this case, the minimization problem is

$\mathbf{j}=\operatorname{minarg}\left\{\left\|L_{T} \mathbf{j}-\mathbf{m}\right\|_{2}^{2}+\lambda^{2}\|\mathbf{j}\|_{2}^{2}+\mu^{2}\|K \mathbf{j}\|_{2}^{2}\right\}$

- The second problem introduces depth-weighting in the temporal term

$\mathbf{j}=\operatorname{minarg}\left\{\left\|L_{T} \mathbf{j}-\mathbf{m}\right\|_{2}^{2}+\lambda^{2}\|B \mathbf{j}\|_{2}^{2}+\mu^{2}\|K B \mathbf{j}\|_{2}^{2}\right\}$
Here, $B=I_{T} \otimes W$. If we change our variables by $\mathbf{x}=B \mathbf{j}$ we get

$$
\mathbf{x}=\operatorname{minarg}\left\{\left\|L_{T} B^{-1} \mathbf{x}-\mathbf{m}\right\|_{2}^{2}+\lambda^{2}\|\mathbf{x}\|_{2}^{2}+\mu^{2}\|K \mathbf{x}\|_{2}^{2}\right\} .
$$

Here, we introduced the depth-weighting of the temporal term because of technical reasons: it makes the transformation above feasible. So we get a minimization problem with the same model-terms as in the first problem (11) above.

The question whether the solutions of these problems are useful has to be decided by practical experiments. At least these solutions should serve as good starting points for the cg-solution of problem (10).

For further calculations, we give the following properties of the kronecker product; see [26]

$$
\begin{aligned}
(A \otimes B)(C \otimes D) & =(A C) \otimes(B D) \\
A \otimes(B+C) & =A \otimes B+A \otimes C .
\end{aligned}
$$

For the sake of simplicity, we show how to solve the first problem given above. In order to tackle the second problem, we will have to replace $L_{T}$ by $L_{T} B^{-1}=I_{T} \otimes\left(L W^{-1}\right)$ in the following calculations. We set $n$ as the number of sensors.

Analogical to (10) the solution of (11) is given by

$$
\left(L_{T}^{\top} L_{T}+\lambda^{2} I_{3 N T}+\mu^{2} K^{\top} K\right) \mathbf{j}=L_{T}^{\top} \mathbf{m} .
$$

Due to the size of $L_{T}^{\top} L_{T}$ the solution of problem (14) is quite expensive. We will see below that we can get a solution by solving a matrix equation involving $L_{T} L_{T}^{\top}$ instead, which only has size $n T \times n T$ and, thus, is much smaller.

Lemma 3: The solution of problem (11) can be achieved by solving

$$
\left(L_{T} L_{T}^{\top}+\lambda^{2} I_{n T}+\mu^{2}\left(D^{\top} D\right) \otimes I_{n}\right) \mathbf{u}=\mathbf{m}
$$

and setting

$$
\mathbf{j}=L_{T}^{\top} \mathbf{u}
$$

Proof: First we see

$$
\begin{aligned}
K^{\top} K L_{T}^{\top} & =\left(\left(D^{\top} D\right) \otimes\left(I_{3 N}\right)\right)\left(I_{T} \otimes L^{\top}\right) \\
& =\left(D^{\top} D\right) \otimes L^{\top} \\
& =\left(I_{T} \otimes L^{\top}\right)\left(\left(D^{\top} D\right) \otimes I_{n}\right) \\
& =L_{T}^{\top} \otimes\left(\left(D^{\top} D\right) \otimes I_{n}\right) .
\end{aligned}
$$

We get

$$
\begin{aligned}
& \left(L_{T}^{\top} L_{T}+\lambda^{2} I_{n T}+\mu^{2} K^{\top} K\right)^{-1} L_{T}^{\top} \\
& \quad=L_{T}^{\top}\left(L_{T} L_{T}^{\top}+\lambda^{2} I_{3 N T}+\mu^{2}\left(D^{\top} D\right) \otimes I_{n}\right)^{-1}
\end{aligned}
$$

by multiplying both sides with the terms in brackets. If we now consider the normal-equation (14) the prove is complete. 
1

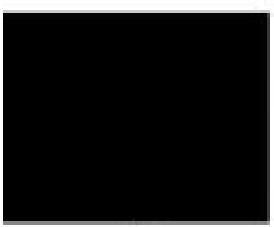

5

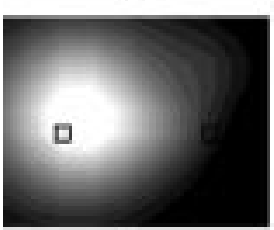

9

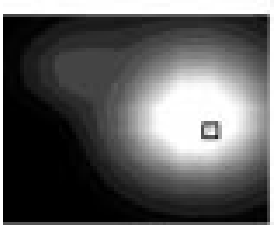

13

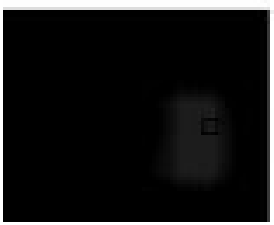

2

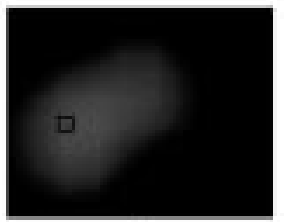

6

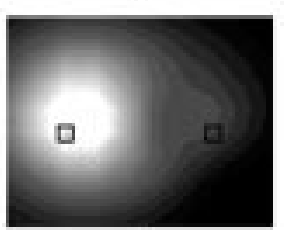

10

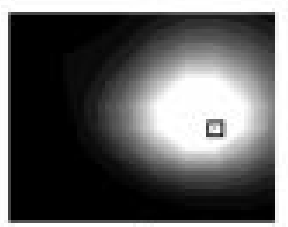

14

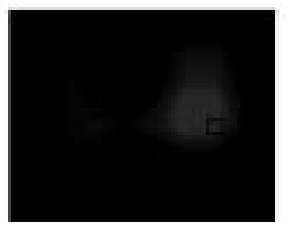

3

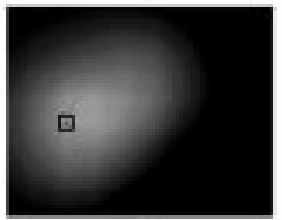

7

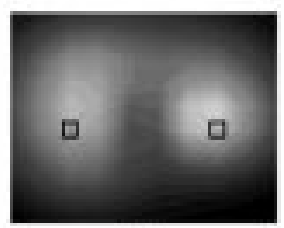

11

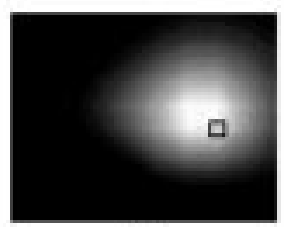

15

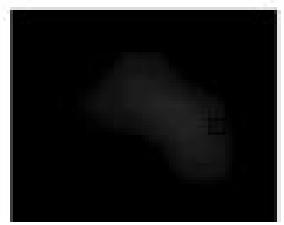

4

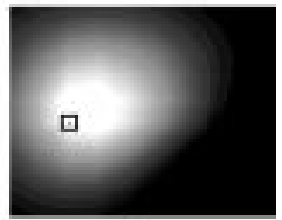

8

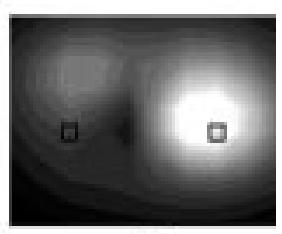

12

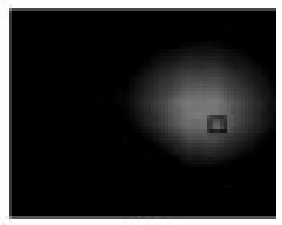

16

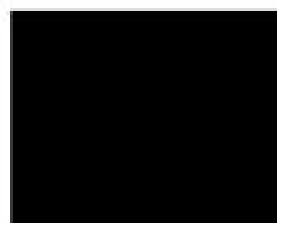

Fig. 16. Temporal reconstruction: zeroth-order spatial smoothness without temporal coupling. Data smoothed with Savitzky-Golay filter. $\lambda^{2}=2.0$. Thirty percent noise added to the data.

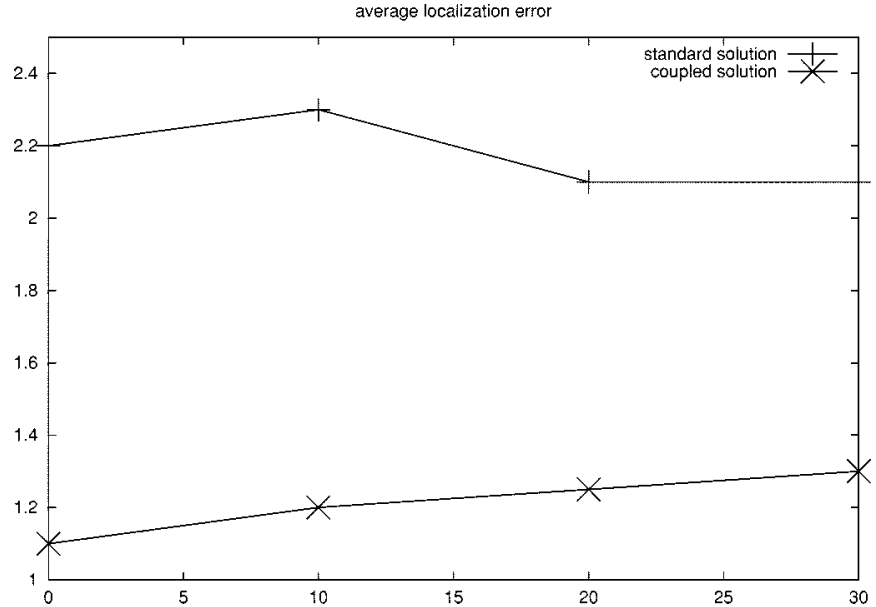

Fig. 17. Spatial accuracy of temporal coupled and noncoupled reconstructions.

Further speedup can be achieved if we consider the Lemma below. We introduce the following notion: If $x$ is a vector of size $m T$ we get $X$ as a matrix of size $m \times T$ by arranging the elements columnwise. We will use this transformation for $y, u, m$ below.

Lemma 4: The solution of $(A \otimes B) x=y$ is equivalent to solving $B X A^{\top}=Y$.

Proof: See [26].

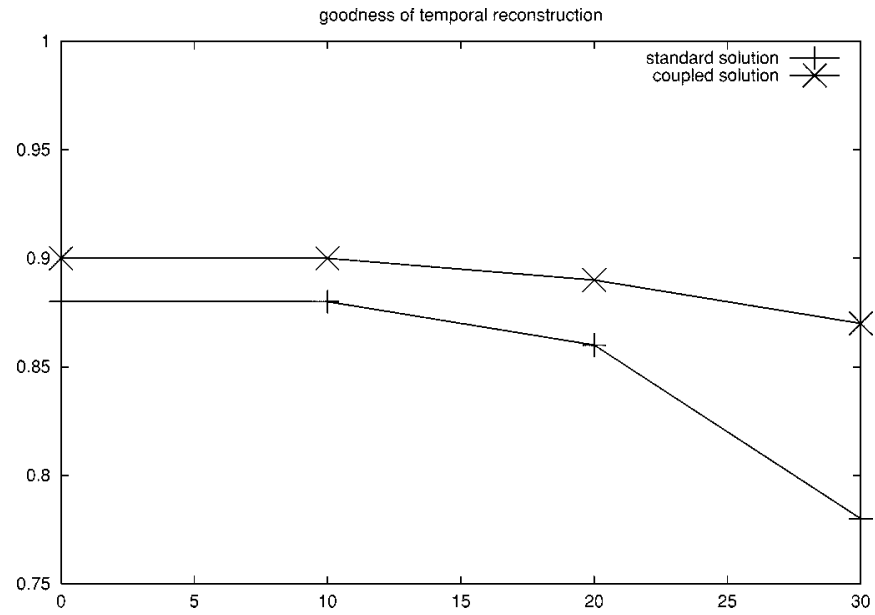

Fig. 18. Temporal accuracy of temporal coupled and noncoupled reconstructions.

Thus, (15) leads to

$$
\left(L L^{\top}+\lambda^{2} I_{n}\right) U+U D^{\top} D=M
$$

with respect to $U$.

This equation of type $A X+X B=C$ is called "continous time" sylvester equation. It can be solved very fast by methods introduced in [27]. 
In some experiments with big influence spaces, the method described above is faster than the direct solution of (14) by a factor of 100 .

The calculations above are not feasible if a matrix $B \neq I$ is present in the spatial model term as in problem (9).

This approach has to be further studied.

\section{REFERENCES}

[1] T. D. Waberski, H. Buchner, G. Herrendorf, R. Gobbel, and W. Paulus, "Properties of inverse methods in temporal lobe epilepsie," Epilepsia, 2000 , to be published.

[2] G. Herrendorf, B. J. Steinhoff, R. Kolle, J. Baudewig, T. D. Waberski, H. Buchner, and W. Paulus, "Dipole source analysis in a realistic head model in patients with focal epilepsy," Epilepsia, no. 41, pp. 71-80, 2000.

[3] H. Buchner, T. D. Waberski, and J. Noth, "Generators of early cortical somatosensory evoked potentials in men," in Recent Advances in Clinical Neurophysiology. Amsterdam, The Netherlands: Elsevier, 1996, pp. 630-636.

[4] H. Buchner, F. Darvas, A. Friedrich, M. Fuchs, A. Knepper, G. Knoll, B. Meyer-Ebrecht, and A. Rienaecker, "Localization of the electromagnetic activity of the brain in its individual anatomy," Future Generation Comput. Syst., 2000.

[5] R. M. Gulrajani, F. A. Roberge, and P. Savard, "The inverse problem of electrocardiography," in Comprehensive Electrocardiol., Macfarlane and V. Lawrie, Eds. New York: Pergamon, 1989, pp. 237-288.

[6] C. Wolters, R. Beckmann, A. Rienaecker, and H. Buchner, "Comparing regularized and nonregularized nonlinear dipole fit methods: A study in a simulated sulcus structure," Brain Topogr., vol. 12, no. 1, pp. 3-18, 1999.

[7] M. Fuchs, M. Wagner, H.-A. Wischmann, T. Köhler, R. Drenckhahn, and H. Buchner, "Improving source reconstructions by combining bioelectric and biomagnetic data," Clin. Neurophysiol., no. 107, pp. 93-111, 1998.

[8] J. Z. Wang, S. J. Willioamson, and L. Kaufmann, "Magnetic source imaging based on the minimum-norm least-squares inverse," Brain Topogr., no. 5, pp. 365-371, 1993.

[9] M. S. Haemaelinen and R. J. Ilmoniemi, "Interpreting magnetic fields of the brain: Minimum norm estimates," Med. Biol. Eng. Comput., no. 32, pp. 35-42, 1994.
[10] M. Fuchs, M. Wagner, T. Köhler, and H.-A. Wischmann, "Linear and nonlinear current density reconstructions," Clin. Neurophysiol., vol. 16, no. 3, pp. 267-295, 1999.

[11] G. Maz'ja, Sobolev Spaces. Berlin, Germany: Springer, 1985.

[12] J.-P. Aubin, Applied Functional Analysis. New York: Wiley, 1999.

[13] W. Hackbusch, Theorie und Numerik elliptischer Differentialgleichungen Stuttgart, Germany, 1986.

[14] H. Buchner, G. Knoll, M. Fuchs, A. Rienaecker, R Beckmann, M. Wagner, J. Silny, and J. Pesch, "Inverse localization of electric dipole current sources in finite element models of the human head," Electroencephalogr. Clin. Neurophysiol., no. 102, pp. 267-278, 1997.

[15] M. Fuchs, R. Drenckhahn, H.-A. Wischmann, and M. Wagner, “An improved boundary element method for realistic volume-conductor modeling," IEEE Trans. Biomed. Eng., vol. 45, pp. 980-997, Aug. 1998.

[16] R. Srebro, "Continuous current source inversion of evoked potential fields in a spherical model head," IEEE Trans. Biomed. Eng., vol. 42, pp. 997-1003, 1994.

[17] A. K. Louis, "Parametric reconstruction in biomagnetic imaging," Inverse Problems in Scattering and Imaging, 1992.

[18] — Inverse und Schlecht Gestellte Probleme Stuttgart, Germany, 1989.

[19] H. W. Engl, M. Hanke, and A. Neubauer, Regularization of Inverse Problems. New York: Kluwer Academic, 1996.

[20] J. P. Kaipio, V. Kolehmainen, E. Somersalo, and M. Vaukhonen, "Statistical inversion and monte carlo sampling methods in electrical impedance tomography," Inverse Problems, no. 16, pp. 1487-1522, 2000.

[21] R. D. Pascual-Marqui, "Low resolution electromagnetic tomography: A new method for localizing electrical activity in brain," Int. J. Psychophysiol., pp. 49-65, 1994.

[22] M. Wagner, "Rekonstruktion Neuronaler Stroeme aus Bioelektrischen Messungen auf der aus MR-Bildern Segmentierten Hirnrinde," masters thesis, Shaker, Germany, 1998.

[23] P. C. Hansen, "Regularization tools," Numer. Algorithms, no. 6, pp. $1-35,1994$.

[24] A Savitzky and M. J. E. Golay, Analytical Chem., vol. 36, pp. $1627-1639,1964$.

[25] R. W. Hamming, Digital Filters, 2nd ed. Englewood Cliffs, NJ: Prentice-Hall, 1983

[26] G. H. Golub and C. F. Van Loan, Matrix Computations, 3rd ed. Baltimore, MD: John Hopkins Univ. Press, 1996.

[27] J. D. Gardiner, A. J. Laub, J. J. Amato, and C. B. Moler, "Solution of the sylvester matrix equation $A X B^{\top}+C X D^{\top}=E$," ACM Trans. Math. Software, vol. 18, no. 2, pp. 223-238, 1992 